\title{
PARTIAL TRANSFORMATION MONOIDS PRESERVING A UNIFORM PARTITION
}

\author{
SERENA CICALÒ, VÍTOR H. FERNANDES AND CSABA SCHNEIDER
}

\begin{abstract}
The objective of this paper is to study the monoid of all partial transformations of a finite set that preserve a uniform partition. In addition to proving that this monoid is a quotient of a wreath product with respect to a congruence relation, we show that it is generated by 5 generators, we compute its order and determine a presentation on a minimal generating set.
\end{abstract}

\section{INTRODUCTION}

The main objective of this paper is a study of the monoid of all partial transformations of a finite set that preserve a uniform equivalence relation. Given a set $\Omega$, a partial transformation on $\Omega$ is a map $\alpha: \Gamma \rightarrow \Omega$ where $\Gamma \subseteq \Omega$ allowing the case that $\Gamma=\emptyset$. The set of partial transformations of $\Omega$ is a monoid under the operation of composition. Given an equivalence relation $E$ on $\Omega$, the set of partial transformations that preserve $E$ is closed under composition, and hence it is a submonoid. In this paper we will investigate monoids of partial transformations preserving a uniform equivalence relation $E$; that is, an equivalence relation with the property that the equivalence classes have the same size. For $n \geqslant 1$, the symbols $\mathcal{P} \mathcal{T}_{n}$ and $\mathcal{T}_{n}$ denote the monoid of all partial transformations and the monoid of all transformations of the set $\underline{n}=\{1, \ldots, n\}$. The wreath product of two monoids $T$ and $S$ is denoted by $T\{S$ (see Section 2 for the definition). The rank of a monoid is the cardinality of a least-order generating set.

The main results of this paper are collected in the following theorem.

Theorem 1.1. Let $\Omega$ be a finite set, let $E$ be a uniform equivalence relation on $\Omega$ with $m$ equivalence classes each of which has size $n$, with $n, m \geqslant 2$, and let $\mathcal{P} \mathcal{T}_{E}$ be the monoid of all partial transformations on $\Omega$ that preserve $E$. Then

(i) $\left|\mathcal{P} \mathcal{T}_{E}\right|=\left(m(n+1)^{n}-m+1\right)^{m}$;

(ii) $\operatorname{rank} \mathcal{P} \mathcal{T}_{E}=5$;

(iii) $\mathcal{P} \mathcal{T}_{E} \cong\left(\mathcal{P} \mathcal{T}_{n} \prec \mathcal{T}_{m}\right) / R$ where $R$ is a congruence relation on $\mathcal{P} \mathcal{T}_{n} \prec \mathcal{T}_{m}$ generated by a single pair of elements.

2000 Mathematics Subject Classification. 20M20, 20M10, 20M05, 05A18.

Key words and phrases. Keywords: Transformation monoids, uniform partitions, ranks, presentations. 
Parts (i) and (iii) of Theorem 1.1 are proved in Section 3, while part (ii) is verified in Section 4. In addition to proving the assertions in Theorem 1.1, we determine an explicit generating set for $\mathcal{P} \mathcal{T}_{E}$ with 5 generators (Corollary 4.3) and a presentation for $\mathcal{P} \mathcal{T}_{E}$ satisfied by this generating set (Corollary 5.3).

The rank of a semigroup is an important invariant and the determination of the ranks of semigroups and monoids has been a focus of research in semigroup theory. Let $\Omega$ be a finite set with at least 2 elements. It is well-known that the full symmetric group of $\Omega$ is generated by 2 generators. (In the language of semigroup theory one might say that the rank of the symmetric group is 2 , but, as the rank has a different meaning in permutation group theory, we avoid expressing this result this way.) Further, the monoid of all transformations and the monoid of all partial transformations of $\Omega$ have ranks 3 and 4, respectively; see [GM09, Theorems 3.1.3 and 3.1.5]. The survey [Fer02] collects these results and similar ones for other classes of transformation monoids, in particular, for monoids of order-preserving transformations and for some of their extensions.

Let $\mathcal{T}_{E}$ denote the stabilizer of a non-trivial uniform equivalence relation $E$ in the monoid of transformations of a finite set $\Omega$. Huisheng [Hui05] proved that $\mathcal{T}_{E}$ is generated by its group of invertible transformations and two additional elements. In other words, the relative rank of $\mathcal{T}_{E}$ with respect to its group of units is 2 . The group of units of $\mathcal{T}_{E}$ is isomorphic to a wreath product of two full symmetric groups and Huisheng observed that such a group can be generated by 4 elements. This led him to conclude that rank $\mathcal{T}_{E} \leqslant 6$. Later, Araújo and the third author AS09. improved this result by showing that the wreath product of two symmetric groups can always be generated by two elements, and hence rank $\mathcal{T}_{E}=4$. Some natural submonoids of $\mathcal{T}_{E}$, such as those of the order-preserving or orientation-preserving transformations, have already been considered in [FQ12, HD05, SPC07, and their ranks were determined by the second author and Quinteiro [FQ11, FQ].

The wreath product construction in the class of monoids is one of the main tools of this paper. The full stabilizer in the symmetric group of a uniform equivalence relation is a wreath product of two smaller symmetric groups and the analogous result holds in the full transformation monoid (see [AS09, Theorem 2.1]). One would expect that the monoid $\mathcal{P} \mathcal{T}_{E}$ of all partial transformations preserving a uniform equivalence relation $E$ is a wreath product of two partial transformation monoids, but this is not the case. Nevertheless, $\mathcal{P} \mathcal{T}_{E}$ can still be described in terms of wreath products. More precisely, $\mathcal{P} \mathcal{T}_{E}$ is isomorphic to a quotient of the wreath product $\mathcal{P} \mathcal{T}_{n} \prec \mathcal{T}_{m}$ where $n$ is the size of an equivalence class and $m$ is the number of classes of $E$. This quotient is taken modulo a well-described congruence relation $R$ (see Lemma 3.1 for details). As claimed by Theorem 1.1(iii), the congruence $R$ is generated by a single pair of elements.

The last part of the paper is concerned with determining a presentation of the monoid $\mathcal{P} \mathcal{T}_{E}$ defined in the previous paragraph. Determining presentations of important semigroups, monoids, and groups has been a mainstream research topic since the end of the 19-th century. For instance, a presentation for the symmetric group $\mathcal{S}_{n}$ was given by 
Moore Moo96 in 1897. For the full transformation monoid $\mathcal{T}_{n}$, a presentation was given by Aǐzenštat Aü58 in 1958 and, some years later, in 1961, Popova Pop62 established a presentation for the partial transformation monoid $\mathcal{P} \mathcal{T}_{n}$. Over the past decades, several authors determined presentations for many other classes of monoids; see Ruš95. and the survey Fer02 on presentations of monoids related to order-preserving transformations. Finally we must mention the recent work of East and his collaborators Eas06, EEF08, Eas10b, Eas10a, Eas11b, Eas11a.

The paper is organized as follows. We set our notation concerning transformations, partial transformations and wreath products in Section 2. We establish the relation between $\mathcal{P} \mathcal{T}_{E}$ and wreath products and prove parts (i) and (iii) of Theorem 1.1 in Section 3, Then in Sections 4 we verify Theorem 1.1(ii). Finally in Section 5 we determine a presentation of $\mathcal{P} \mathcal{T}_{E}$ on a minimal generating set.

\section{NOTATION CONCERNING PARTIAL TRANSFORMATIONS AND WREATH PRODUCTS}

In this section we establish the notation concerning partial transformations and wreath products that will be used throughout the paper.

Let $\Omega$ be a finite set. A partial transformation of $\Omega$ is a map $\alpha: \Gamma \rightarrow \Omega$ where $\Gamma \subseteq \Omega$. The set $\Gamma$ is the domain of $\alpha$ and is denoted by $\operatorname{Dom} \alpha$, while the image of $\alpha$ is denoted by $\operatorname{Im} \alpha$. If $\Gamma=\Omega$ then $\alpha$ is said to be a transformation. Sometimes we want to emphasize that a partial transformation is or is not a transformation and in such cases we write full transformation and strictly partial transformation, respectively. We allow the case that $\Gamma=\emptyset$ and the corresponding partial transformation is denoted by $\varnothing$. In this paper, transformations and partial transformations act on the right unless it is explicitly stated otherwise. That is, if $\alpha$ is a partial transformation on $\Omega$ and $i \in \Omega$, then the image of $i$ under $\alpha$ is denoted by $i \alpha$. The set of all partial transformations of $\Omega$ is a monoid under composition and is denoted by $\mathcal{P} \mathcal{T}(\Omega)$. Given an equivalence relation $E$ on $\Omega$ we say that a partial transformation $\alpha$ preserves $E$ if for all $i, j \in \operatorname{Dom} \alpha$ such that $(i, j) \in E$ we have that $(i \alpha, j \alpha) \in E$. The equivalence relation $E$ is said to be uniform if its equivalence classes have the same size. The equivalence relations $\Omega \times \Omega$ and $\{(i, i) \mid i \in \Omega\}$ are preserved by $\mathcal{P} \mathcal{T}(\Omega)$, and these relations are said to be trivial. Hence an equivalence relation is non-trivial if and only if it has at least two classes and at least one class has size 2. For a natural number $n$, let $\underline{n}$ denote the set $\{1, \ldots, n\}$. The symbols $\mathcal{P} \mathcal{T}_{n}, \mathcal{T}_{n}$, and $\mathcal{S}_{n}$ denote the monoid of partial transformations, the monoid of transformations, and the group of permutations, respectively, acting on $\underline{n}$.

We represent a transformation $\alpha \in \mathcal{T}_{n}$ by the list $[1 \alpha, \ldots, n \alpha]$ of images. If $\alpha$ is a partial transformation and $i \notin \operatorname{Dom} \alpha$ then we put $\varnothing$ in the place of $i \alpha$. A permutation is usually written as a product of cycles. The identity map in $\mathcal{T}_{n}$ will be written as 1 .

Let us now review the concept of wreath products of monoids; see [RS09, Section 1.2.2] for details. Let $m \in \mathbb{N}$, let $T$ be a submonoid of $\mathcal{T}_{m}$, and let $S$ be any monoid. The wreath 
product of $S$ by the transformation monoid $T$, denoted by $S \nmid T$, is the semidirect product $S^{m} \rtimes T$ with respect to the left action of $T$ on the $m$-fold direct power $S^{m}$ defined as

$$
\tau\left(s_{1}, s_{2}, \ldots, s_{m}\right)=\left(s_{1 \tau}, s_{2 \tau}, \ldots, s_{m \tau}\right),
$$

for all $\tau \in T$ and $s_{1}, s_{2}, \ldots, s_{m} \in S$. Therefore, the wreath product $S 々 T$ is the monoid with underlying set $S^{m} \times T$ and multiplication defined by

$$
\begin{array}{r}
\left(s_{1}, s_{2}, \ldots, s_{m} ; \sigma\right)\left(t_{1}, t_{2}, \ldots, t_{m} ; \tau\right)=\left(\left(s_{1}, s_{2}, \ldots, s_{m}\right)^{\sigma}\left(t_{1}, t_{2}, \ldots, t_{m}\right) ; \sigma \tau\right)= \\
\left(s_{1} t_{1 \sigma}, s_{2} t_{2 \sigma}, \ldots, s_{m} t_{m \sigma} ; \sigma \tau\right)
\end{array}
$$

for all $\left(s_{1}, s_{2}, \ldots, s_{m} ; \sigma\right),\left(t_{1}, t_{2}, \ldots, t_{m} ; \tau\right) \in S^{m} \times T$.

An element $\beta$ of a wreath product $S<T$ can be written uniquely in the form $\left(\beta^{(1)}, \ldots, \beta^{(m)} ; \bar{\beta}\right)$ where $\beta^{(1)}, \ldots, \beta^{(m)} \in S$ and $\bar{\beta} \in T$. In the rest of the paper, for an element $\beta \in S \imath T$, these components of $\beta$ will be denoted by $\beta^{(1)}, \ldots, \beta^{(m)}, \bar{\beta}$.

\section{WREATH PRODUCTS AND PARTIAL ENDOMORPHISMS OF A UNIFORM PARTITION}

Let $m, n \in \mathbb{N}$ and set $\Omega=\underline{n} \times \underline{m}$. Let $E$ denote the equivalence relation on $\Omega$ that is defined by the rule that $((i, j),(k, l)) \in E$ if and only if $j=l$. Then $E$ has $m$ equivalence classes each of which has size $n$, and hence $E$ is uniform. Let $\mathcal{P} \mathcal{T}_{n \times m}, \mathcal{T}_{n \times m}$, and $\mathcal{S}_{n \times m}$ denote the monoid of all partial transformations, the monoid of all transformations, and the group of all permutations on $\Omega$ preserving the equivalence relation $E$. An isomorphism between $\mathcal{T}_{n \times m}$ and the wreath product $\mathcal{T}_{n}<\mathcal{T}_{m}$ can easily be constructed as follows (see [AS09, Lemma 2.1]). Let $\alpha \in \mathcal{T}_{n \times m}$. The transformation $\alpha$ induces a transformation $\bar{\alpha}$ on the set of equivalence classes of $E$. Since these equivalence classes are indexed by the elements of $\underline{m}$, the transformation $\bar{\alpha}$ can be viewed as an element of $\mathcal{T}_{m}$. Further, for each $j \in \underline{m}$, we can define a transformation $\alpha^{(j)}$ on $\underline{n}$ as follows: $i \alpha^{(j)}=k$ if $(i, j) \alpha=(k, j \bar{\alpha})$. It is proved in AS09, Lemma 2.1] that the map $\alpha \mapsto\left(\alpha^{(1)}, \ldots, \alpha^{(m)} ; \bar{\alpha}\right)$ is an isomorphism between the monoids $\mathcal{T}_{n \times m}$ and $\mathcal{T}_{n} \prec \mathcal{T}_{m}$.

One would think that the monoids $\mathcal{P} \mathcal{T}_{n \times m}$ may be isomorphic to a wreath product constructed from $\mathcal{P} \mathcal{T}_{n}$ and $\mathcal{T}_{m}$, but unfortunately this is not the case. Nonetheless, the monoid $\mathcal{P} \mathcal{T}_{n \times m}$ can be described as a quotient of the wreath product $\mathcal{P} \mathcal{T}_{n} \prec \mathcal{T}_{m}$ as follows. We define a homomorphism $\varphi$ from $\mathcal{P} \mathcal{T}_{n} \prec \mathcal{T}_{m}$ to $\mathcal{P} \mathcal{T}_{n \times m}$ by defining a "partial action" of $\mathcal{P} \mathcal{T}_{n} \prec \mathcal{T}_{m}$ on $\Omega=\underline{n} \times \underline{m}$. Let $\alpha \in \mathcal{P} \mathcal{T}_{n} \succ \mathcal{T}_{m}$. We define $\alpha \varphi \in \mathcal{P} \mathcal{T}_{n \times m}$ as follows. Let

$$
\operatorname{Dom} \alpha \varphi=\left\{(i, j) \mid i \in \operatorname{Dom} \alpha^{(j)}\right\}
$$

and for $(i, j) \in \operatorname{Dom} \alpha \varphi$ we set $(i, j)(\alpha \varphi)=\left(i \alpha^{(j)}, j \bar{\alpha}\right)$. It is routine calculation to check that $\varphi: \mathcal{P} \mathcal{T}_{n} \prec \mathcal{T}_{m} \rightarrow \mathcal{P} \mathcal{T}_{n \times m}$ is a homomorphism.

For $j \in \underline{m}$, let $\varepsilon^{(j)}$ denote the homomorphism $\mathcal{P} \mathcal{T}_{n} \rightarrow \mathcal{P} \mathcal{T}_{n} \prec \mathcal{T}_{m}$ defined by the rule $\alpha \mapsto(1, \ldots, 1, \alpha, 1, \ldots, 1 ; 1)$ where the non-trivial factor of the image appears in the $j$-th position. Further, let $\bar{\varepsilon}$ denote the homomorphism $\mathcal{T}_{m} \rightarrow \mathcal{P} \mathcal{T}_{n} \prec \mathcal{T}_{m}$ mapping $\alpha \mapsto(1, \ldots, 1 ; \alpha)$. The homomorphisms $\varepsilon^{(j)}$ and $\bar{\varepsilon}$ are clearly injective. 
Lemma 3.1. (i) The homomorphism $\varphi$ defined above is surjective.

(ii) For $\alpha, \beta \in \mathcal{P} \mathcal{T}_{n} \curlywedge \mathcal{T}_{m}$, we have that $\alpha \varphi=\beta \varphi$ if and only if $\alpha^{(j)}=\beta^{(j)}$ for all $j \in \underline{m}$ and $j \bar{\alpha}=j \bar{\beta}$ whenever $\alpha^{(j)} \neq \varnothing$.

(iii) Let $\tau$ denote the element $[2,2,3, \ldots, m] \bar{\varepsilon}$ of $\mathcal{P} \mathcal{T}_{n} \imath \mathcal{T}_{m}$. Then the congruence relation $\operatorname{Ker} \varphi$ is generated by the pair $\left(\varnothing \varepsilon^{(1)},\left(\varnothing \varepsilon^{(1)}\right) \tau\right)$.

Proof. (i) Let $\alpha \in \mathcal{P} \mathcal{T}_{n \times m}$. We define $\alpha^{(1)}, \ldots, \alpha^{(m)} \in \mathcal{P} \mathcal{T}_{n}$ and $\bar{\alpha} \in \mathcal{T}_{m}$ similarly as in the argument that $\mathcal{T}_{n}<\mathcal{T}_{m} \cong \mathcal{T}_{n \times m}$ before the lemma. The only difference is that, for some $j$, the restriction of $\alpha$ to the equivalence class $\{(i, j) \mid i \in \underline{n}\}$ may be equal to $\varnothing$. In this case we let $j \bar{\alpha}=1$. Then it is clear that $\left(\alpha^{(1)}, \ldots, \alpha^{(m)} ; \bar{\alpha}\right) \varphi=\alpha$, and hence $\varphi$ is surjective.

(ii) Let us first assume that $\alpha, \beta \in \mathcal{P} \mathcal{T}_{n} \prec \mathcal{T}_{m}$ such that $\alpha \varphi=\beta \varphi$. If $\alpha^{(j)} \neq \beta^{(j)}$, for some $j$, then there is $i \in \underline{n}$ such that $i \alpha^{(j)} \neq i \beta^{(j)}$ (including the possibility that either $i \notin \operatorname{Dom} \alpha$ or $i \notin \operatorname{Dom} \beta)$. Then $(i, j)(\alpha \varphi) \neq(i, j)(\beta \varphi)$, which is a contradiction. Similarly, if $j \in \underline{m}$ is such that $\alpha^{(j)} \neq \varnothing$, but $j \bar{\alpha} \neq j \bar{\beta}$, then we have, for $i \in \operatorname{Dom} \alpha^{(j)}$, that $(i, j)(\alpha \varphi) \neq(i, j)(\beta \varphi)$. Therefore we obtain a contradiction again. Thus one direction of assertion (ii) is valid.

To show the other direction, let us now suppose that $\alpha, \beta \in \mathcal{P} \mathcal{T}_{n} \prec \mathcal{T}_{m}$ such that $\alpha^{(j)}=\beta^{(j)}$ for all $j \in \underline{m}$ and $j \bar{\alpha}=j \bar{\beta}$ whenever $\alpha^{(j)} \neq \varnothing$. Equation (3) implies that $\operatorname{Dom}(\alpha \varphi)=\operatorname{Dom}(\beta \varphi)$. Let $(i, j) \in \operatorname{Dom}(\alpha \varphi)$. Then $\alpha^{(j)} \neq \varnothing$ and $\beta^{(j)} \neq \varnothing$ and so

$$
(i, j)(\alpha \varphi)=\left(i \alpha^{(j)}, j \bar{\alpha}\right)=\left(i \beta^{(j)}, j \bar{\beta}\right)=(i, j)(\beta \varphi) .
$$

Hence $\alpha \varphi=\beta \varphi$ as required.

(iii) Let $Q$ be the smallest congruence relation on the monoid $\mathcal{P} \mathcal{T}_{n} \prec \mathcal{T}_{m}$ such that $\left(\varnothing \varepsilon^{(1)},\left(\varnothing \varepsilon^{(1)}\right) \tau\right) \in Q$. By the description of $\operatorname{Ker} \varphi$ in statement (ii), we have that $\left(\varnothing \varepsilon^{(1)},\left(\varnothing \varepsilon^{(1)}\right) \tau\right) \in \operatorname{Ker} \varphi$ and hence $Q \subseteq \operatorname{Ker} \varphi$. It remains to show that $\operatorname{Ker} \varphi \subseteq Q$.

For $i, s \in \underline{m}$, let $\tau_{i, s}$ denote the transformation $[1,2, \ldots, i-1, s, i+1, i+2, \ldots, m]$. We claim that

$$
\left(\varnothing \varepsilon^{(i)},\left(\varnothing \varepsilon^{(i)}\right)\left(\tau_{i, s} \bar{\varepsilon}\right)\right) \in Q \quad \text { for all } i, s \in \underline{m} .
$$

First we verify this claim in the case when $i=1$. Let $s \in\{2, \ldots, m\}$. As

$$
((2 s) \bar{\varepsilon})\left(\varnothing \varepsilon^{(1)}\right)((2 s) \bar{\varepsilon})=\varnothing \varepsilon^{(1)}
$$

while

$$
((2 s) \bar{\varepsilon})\left(\varnothing \varepsilon^{(1)}\right) \tau((2 s) \bar{\varepsilon})=\left(\varnothing \varepsilon^{(1)}\right)\left(\tau_{1, s} \bar{\varepsilon}\right),
$$

we find that $\left(\varnothing \varepsilon^{(1)},\left(\varnothing \varepsilon^{(1)}\right)\left(\tau_{1, s} \bar{\varepsilon}\right)\right) \in Q$. Now conjugating this pair with $(1 i) \bar{\varepsilon}$ gives (41).

Suppose that $\alpha, \beta \in \mathcal{P} \mathcal{T}_{n} \prec \mathcal{T}_{m}$ such that $(\alpha, \beta) \in \operatorname{Ker} \varphi$. We are required to show that $(\alpha, \beta) \in Q$. We have, for all $j \in \underline{m}$, that $\alpha^{(j)}=\beta^{(j)}$ and that $j \bar{\alpha}=j \bar{\beta}$ whenever $\alpha^{(j)} \neq \varnothing$. Let $I=\{j \mid j \in \underline{m}$ with $j \bar{\alpha} \neq \bar{j} \bar{\beta}\}$. We show by induction on $|I|$ that $(\alpha, \beta) \in Q$. If $I=\emptyset$, then $\alpha=\beta$, and in this case the claim is clearly valid. 
Suppose that $I \neq \emptyset$ and that $\left(\alpha_{1}, \beta_{1}\right) \in \operatorname{Ker} \varphi$ implies that $\left(\alpha_{1}, \beta_{1}\right) \in Q$ whenever $\left|\left\{j \mid j \bar{\alpha}_{1} \neq j \bar{\beta}_{1}\right\}\right| \leqslant|I|-1$. Let $j_{0}$ be the minimal element of $I$. After possibly replacing $\alpha$ and $\beta$ with $\left(\left(1 j_{0}\right) \bar{\varepsilon}\right) \alpha$ and $\left(\left(1 j_{0}\right) \bar{\varepsilon}\right) \beta$, we may assume that $j_{0}=1$. Thus we have that $1 \bar{\alpha}=r$ and $1 \bar{\beta}=s$ with $r \neq s$ and that $\alpha^{(1)}=\beta^{(1)}=\varnothing$. Suppose first that $s \in \operatorname{Im} \bar{\alpha}$; that is, $s=u \bar{\alpha}$ with some $u \in \underline{m}$. Then

$$
\left(\varnothing \varepsilon^{(1)}\right) \alpha=\alpha
$$

while

$$
\left(\varnothing \varepsilon^{(1)}\right)\left(\tau_{1, u} \bar{\varepsilon}\right) \alpha=\left(\alpha^{(1)}, \alpha^{(2)}, \ldots, \alpha^{(m)} ; \bar{\alpha}^{\prime}\right)
$$

where $1 \bar{\alpha}^{\prime}=s$ and $j \bar{\alpha}^{\prime}=j \bar{\alpha}$ for all $j \in\{2, \ldots, m\}$. Hence $\left(\alpha,\left(\alpha^{(1)}, \alpha^{(2)}, \ldots, \alpha^{(m)} ; \bar{\alpha}^{\prime}\right)\right) \in Q$. Since $\left|\left\{j \mid j \bar{\alpha}^{\prime} \neq j \bar{\beta}\right\}\right| \leqslant|I|-1$, we obtain from the induction hypothesis that $\left(\beta,\left(\alpha^{(1)}, \alpha^{(2)}, \ldots, \alpha^{(m)} ; \bar{\alpha}^{\prime}\right)\right) \in Q$. Thus $(\alpha, \beta) \in Q$ as claimed.

If $s \notin \operatorname{Im} \bar{\alpha}$, but $r \in \operatorname{Im} \bar{\beta}$ then, interchanging the role of $\alpha$ and $\beta$, the same argument shows that $(\alpha, \beta) \in Q$.

Suppose now that $s \notin \operatorname{Im} \bar{\alpha}$ and $r \notin \operatorname{Im} \bar{\beta}$. If there is some $j \in \underline{m}$ such that $\alpha^{(j)} \neq \varnothing$ then $j \bar{\alpha}=j \bar{\beta}$. Set $u=j \bar{\alpha}$ and let $\bar{\alpha}^{\prime}=[u, 2 \bar{\alpha}, 3 \bar{\alpha}, \ldots, m \bar{\alpha}]$ and $\bar{\beta}^{\prime}=[u, 2 \bar{\beta}, 3 \bar{\beta}, \ldots, m \bar{\beta}]$. By the argument above, we have that $\left(\alpha,\left(\alpha^{(1)}, \ldots, \alpha^{(m)} ; \bar{\alpha}^{\prime}\right)\right) \in Q$ and $\left(\beta,\left(\beta^{(1)}, \ldots, \beta^{(m)} ; \bar{\beta}^{\prime}\right)\right) \in Q$. By the induction hypothesis, $\left(\left(\alpha^{(1)}, \ldots, \alpha^{(m)} ; \bar{\alpha}^{\prime}\right),\left(\beta^{(1)}, \ldots, \beta^{(m)} ; \bar{\beta}^{\prime}\right)\right) \in Q$ which implies that $(\alpha, \beta) \in Q$ in this case.

Finally we prove the claim when $\alpha^{(j)}=\varnothing$ for all $j \in \underline{m}$. In this case we are required to show that $((\varnothing, \ldots, \varnothing ; \bar{\alpha}),(\varnothing, \ldots, \varnothing ; \bar{\beta})) \in Q$ for all $\bar{\alpha}, \bar{\beta} \in \mathcal{T}_{m}$. The elements $\alpha$ with the property that $\alpha^{(j)}=\varnothing$ for all $j \in \underline{m}$ form a submonoid $T$ in $\mathcal{P} \mathcal{T}_{n}\left\langle\mathcal{T}_{m}\right.$ that is isomorphic to $\mathcal{T}_{m}$ via the isomorphism $\alpha \mapsto \bar{\alpha}$. The relation $Q_{T}=Q \cap(T \times T)$ is a congruence relation on $T$. Equation (4) implies that

$$
\left(\prod_{j \in \underline{m}} \varnothing \varepsilon^{(j)}, \prod_{j \in \underline{m}}\left(\varnothing \varepsilon^{(j)}\right)\left(\tau_{j, 1} \bar{\varepsilon}\right)\right) \in Q_{T} .
$$

Now $\prod_{j \in \underline{m}} \varnothing \varepsilon^{(j)}=(\varnothing, \ldots, \varnothing ; 1)$ while $\prod_{j \in \underline{m}}\left(\varnothing \varepsilon^{(j)}\right)\left(\tau_{j, 1} \bar{\varepsilon}\right)=(\varnothing, \ldots, \varnothing ;[1, \ldots, 1])$. Therefore $((\varnothing, \ldots, \varnothing ; 1),(\varnothing, \ldots, \varnothing ;[1, \ldots, 1])) \in Q_{T}$. Now [GM09, Theorem 6.3.10] gives that $Q_{T}$ must be the universal congruence which shows that $((\varnothing, \ldots, \varnothing ; \bar{\alpha}),(\varnothing, \ldots, \varnothing ; \bar{\beta})) \in Q_{T}$ for all $\bar{\alpha}, \bar{\beta} \in \mathcal{T}_{m}$. Therefore $((\varnothing, \ldots, \varnothing ; \bar{\alpha}),(\varnothing, \ldots, \varnothing ; \bar{\beta})) \in Q$ for all $\bar{\alpha}, \bar{\beta} \in \mathcal{T}_{m}$ as required.

Part (iii) of Lemma 3.1 implies Theorem 1.1(iii).

Next we determine the order of $\mathcal{P} \mathcal{T}_{n \times m}$ by counting the equivalence classes of the relation $\operatorname{Ker} \varphi$ defined in Lemma 3.1. Let $\alpha \in \mathcal{P} \mathcal{T}_{n} \prec \mathcal{T}_{m}$. We say that $\alpha$ is in canonical form if $\alpha^{(j)}=\varnothing$ implies that $j \bar{\alpha}=1$ for all $j \in \underline{m}$. 
Lemma 3.2. Every equivalence class of $\operatorname{Ker} \varphi$ contains precisely one element in canonical form. Consequently,

$$
\left|\mathcal{P} \mathcal{T}_{n \times m}\right|=\left(m(n+1)^{n}-m+1\right)^{m}
$$

Proof. Let $\alpha \in \mathcal{P} \mathcal{T}_{n} \prec \mathcal{T}_{m}$. Then $\alpha=\left(\alpha^{(1)}, \ldots, \alpha^{(m)} ; \bar{\alpha}\right)$ where $\alpha^{(1)}, \ldots, \alpha^{(m)} \in \mathcal{P} \mathcal{T}_{n}$ and $\bar{\alpha}=\mathcal{T}_{m}$. For $j \in \underline{m}$, set $\beta^{(j)}=\alpha^{(j)}$ and define $\bar{\beta} \in \mathcal{T}_{m}$ by the rule that $j \bar{\beta}=j \bar{\alpha}$ if $\alpha^{(j)} \neq \varnothing$ and $j \bar{\beta}=1$ otherwise. Then the element $\beta=\left(\beta^{(1)}, \ldots, \beta^{(m)} ; \bar{\beta}\right)$ is in canonical form and $\alpha \varphi=\beta \varphi$. Hence every congruence class of $\operatorname{Ker} \varphi$ contains an element in canonical form.

Assume that $\alpha, \beta \in \mathcal{P} \mathcal{T}_{n} \succ \mathcal{T}_{m}$ are in canonical form such that $\alpha \varphi=\beta \varphi$. We have by Lemma 3.1 that $\alpha^{(j)}=\beta^{(j)}$ for all $j \in \underline{m}$ and that $j \bar{\alpha}=j \bar{\beta}$ for all $j \in \underline{m}$ such that $\alpha^{(j)} \neq \varnothing$. On the other hand, if $\alpha^{(j)}=\varnothing$ then, as $\alpha$ and $\beta$ are in canonical form, $j \bar{\alpha}=j \bar{\beta}=1$. Hence we obtain that $\alpha=\beta$ and so each of the congruence classes contains precisely one element in canonical form.

To compute the order of $\mathcal{P} \mathcal{T}_{n \times m}$, let us compute the number of elements in canonical form. For $r \in \underline{m}$, let $A_{r}$ denote the number of elements $\alpha$ in canonical form such that $\left|\left\{j \in \underline{m} \mid \alpha^{(j)} \neq \varnothing\right\}\right|=r$. Then

$$
A_{r}=\left(\begin{array}{c}
m \\
r
\end{array}\right)\left(\left|\mathcal{P} \mathcal{T}_{n}\right|-1\right)^{r} m^{r}
$$

Thus

$\left|\mathcal{P} \mathcal{T}_{n \times m}\right|=\sum_{r=0}^{m}\left(\begin{array}{c}m \\ r\end{array}\right)\left(\left|\mathcal{P} \mathcal{T}_{n}\right|-1\right)^{r} m^{r}=\sum_{r=0}^{m}\left(\begin{array}{c}m \\ r\end{array}\right)\left(m(n+1)^{n}-m\right)^{r}=\left(m(n+1)^{n}-m+1\right)^{m}$,

as claimed.

The second assertion of Lemma 3.2 implies Theorem 1.1(i). The table below shows the order of the monoid $\mathcal{P} \mathcal{T}_{n \times m}$ for small values of $m$ and $n$.

\begin{tabular}{r|rrrrr}
$m$ & $\left|\mathcal{P} \mathcal{T}_{m}\right|$ & $\left|\mathcal{P} \mathcal{T}_{2 \times m}\right|$ & $\left|\mathcal{P} \mathcal{T}_{3 \times m}\right|$ & $\left|\mathcal{P} \mathcal{T}_{4 \times m}\right|$ & $\left|\mathcal{P} \mathcal{T}_{5 \times m}\right|$ \\
\hline 1 & 2 & 9 & 64 & 625 & 7776 \\
2 & 9 & 289 & 16129 & 1560001 & 115856201 \\
3 & 64 & 15625 & 6859000 & 6570725617 & 3150905752576 \\
4 & 625 & 1185921 & 4097152081 & 38875337230081 & 296120751810639601 \\
5 & 7776 & 115856201 & 3150905752576 & 296120751810639601 & 88798957515761812069376
\end{tabular}

\section{THE RANK}

In this section we determine the ranks of $\mathcal{P} \mathcal{T}_{n \times m}$ and $\mathcal{P} \mathcal{T}_{n} \prec \mathcal{T}_{m}$ defined in Section 3 ,

Theorem 4.1. If $m \geq 2$ and $n \geq 2$, then $\operatorname{rank} \mathcal{P} \mathcal{T}_{n} \curlywedge \mathcal{T}_{m}=\operatorname{rank} \mathcal{P} \mathcal{T}_{n \times m}=5$. 
Before proving the theorem, we review some known facts about the ranks and generating sets of the monoids $\mathcal{P} \mathcal{T}_{n}, \mathcal{T}_{n}, \mathcal{T}_{n} \imath \mathcal{T}_{m}$ and fix some notation. Let us consider the following elements of $\mathcal{T}_{n} \prec \mathcal{T}_{m}$ :

$$
\begin{aligned}
& \xi_{1}= \begin{cases}\left((12) \varepsilon^{(2)}\right)((23 \cdots m) \bar{\varepsilon}) & \text { if } m \text { and } n \text { are both even } \\
\left((12) \varepsilon^{(2)}\right)\left(\left(\begin{array}{lll}
1 & 2 \cdots m) \bar{\varepsilon}) & \text { otherwise; }
\end{array}\right.\right.\end{cases}
\end{aligned}
$$

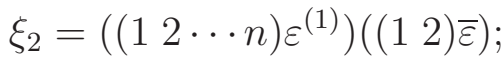

$$
\begin{aligned}
& \tau=[2,2,3, \ldots, n] \varepsilon^{(1)} \\
& \bar{\tau}=[2,2,3, \ldots, m] \bar{\varepsilon} \text {. }
\end{aligned}
$$

Then the following lemma is valid.

Lemma 4.2. (i) If $\gamma \in \mathcal{P} \mathcal{T}_{n}$ then $\mathcal{P} \mathcal{T}_{n}=\left\langle\mathcal{T}_{n}, \gamma\right\rangle$ if and only if $\gamma \in \mathcal{P} \mathcal{T}_{n} \backslash \mathcal{T}_{n}$ and rank $\gamma=n-1$.

(ii) We have that $\operatorname{rank} \mathcal{S}_{n} \curlywedge \mathcal{S}_{m}=2$ and $\operatorname{rank} \mathcal{T}_{n} \curlywedge \mathcal{T}_{m}=4$. Further, $\mathcal{S}_{n} \curlywedge \mathcal{S}_{m}=\left\langle\xi_{1}, \xi_{2}\right\rangle$ and $\mathcal{T}_{n} \prec \mathcal{T}_{m}=\left\langle\xi_{1}, \xi_{2}, \tau, \bar{\tau}\right\rangle$.

(iii) Let $P$ be a monoid of partial transformations of a finite set and let $G$ be the group of permutations in $P$. If $X$ is a generating set of $P$ then $X \cap G$ is a generating set of $G$.

Proof. Statement (i) follows from [GM09, Theorem 3.1.5]. Combining AS09, Theorem 4.1, Lemma 3.2, and Theorem 1.1] gives statement (ii). (Note that the generators that correspond to $\tau$ and $\bar{\tau}$ appear in [AS09] in a slightly different form.) Finally statement (iii) can be easily proved using the argument in [AS09, Lemma 3.1].

Now we can prove Theorem 4.1 .

Proof of Theorem 4.1. First we show that the monoids $\mathcal{P} \mathcal{T}_{n \times m}$ and $\mathcal{P} \mathcal{T}_{n} 々 \mathcal{T}_{m}$ are generated by 5 elements. Since, by Lemma 3.1. $\mathcal{P} \mathcal{T}_{n \times m}$ is a homomorphic image of $\mathcal{P} \mathcal{T}_{n} \imath \mathcal{T}_{m}$, it suffices to show that $\mathcal{P} \mathcal{T}_{n} \prec \mathcal{T}_{m}$ is generated by 5 elements. Let $\sigma_{1}=[\varnothing, 2,3, \ldots, n]$; then, by Lemma 4.2(i), $\mathcal{P} \mathcal{T}_{n}=\left\langle\mathcal{T}_{n}, \sigma_{1}\right\rangle$. Set $\sigma=\sigma_{1} \varepsilon^{(1)}=\left(\sigma_{1}, 1, \ldots, 1 ; 1\right)$. We claim that $\mathcal{P} \mathcal{T}_{n}\left\langle\mathcal{T}_{m}=\left\langle\mathcal{T}_{n} \imath \mathcal{T}_{m}, \sigma\right\rangle\right.$. Once this assertion is proved, the claim that $\mathcal{P} \mathcal{T}_{n}\left\langle\mathcal{T}_{m}\right.$ is generated by 5 elements will follow, as $\operatorname{rank} \mathcal{T}_{n} \prec \mathcal{T}_{m}=4$ (Lemma $4.2($ ii) $)$.

Let us now prove that $\mathcal{P} \mathcal{T}_{n} \imath \mathcal{T}_{m}=\left\langle\mathcal{T}_{n} \imath \mathcal{T}_{m}, \sigma\right\rangle$. Set $W=\left\langle\mathcal{T}_{n} \imath \mathcal{T}_{m}, \sigma\right\rangle$. First we note that $\mathcal{T}_{m} \bar{\varepsilon} \leqslant W$. The fact that $\mathcal{P} \mathcal{T}_{n}=\left\langle\mathcal{T}_{n}, \sigma_{1}\right\rangle$ implies that $\mathcal{P} \mathcal{T}_{n} \varepsilon^{(1)}=\left\langle\mathcal{T}_{n} \varepsilon^{(1)}, \sigma\right\rangle$. As $\mathcal{T}_{n} \varepsilon^{(1)} \leqslant W$, we obtain that $\mathcal{P} \mathcal{T}_{n} \varepsilon^{(1)} \leqslant W$. For $j \geqslant 1$, it is a consequence of equation (2) that $((1 j) \bar{\varepsilon})\left(\mathcal{P} \mathcal{T}_{n} \varepsilon^{(1)}\right)((1 j) \bar{\varepsilon})=\mathcal{P} \mathcal{T}_{n} \varepsilon^{(j)}$. As $\mathcal{P} \mathcal{T}_{n} \varepsilon^{(1)} \leqslant W$ and $\mathcal{S}_{m} \bar{\varepsilon} \leqslant W$, we find that $\mathcal{P} \mathcal{T}_{n} \varepsilon^{(j)} \leqslant W$ for all $j \in \underline{m}$. Since the monoids $\mathcal{T}_{m} \bar{\varepsilon}$ and $\mathcal{P} \mathcal{T}_{n} \varepsilon^{(j)}$ with $j \in \underline{m}$ are contained in $W$ and they generate $\overline{\mathcal{P}} \mathcal{T}_{n} \prec \mathcal{T}_{m}$, we obtain that $\mathcal{P} \mathcal{T}_{n} \imath \mathcal{T}_{m} \leqslant W$. As clearly, $W \leqslant \mathcal{P} \mathcal{T}_{n} \iota \mathcal{T}_{m}$ we conclude that $\mathcal{P} \mathcal{T}_{n} \prec \mathcal{T}_{m}=W$, as claimed.

Next we show that the monoids $\mathcal{P} \mathcal{T}_{n \times m}$ and $\mathcal{P} \mathcal{T}_{n} \prec \mathcal{T}_{m}$ cannot be generated by fewer than 5 elements. We note, as above, that $\mathcal{P} \mathcal{T}_{n \times m}$ is a homomorphic image of $\mathcal{P} \mathcal{T}_{n} \imath \mathcal{T}_{m}$, 
and we need only prove this for $\mathcal{P} \mathcal{T}_{n \times m}$. Let $G$ denote the unit group of $\mathcal{P} \mathcal{T}_{n \times m}$. Let $X$ be a subset of $\mathcal{P} \mathcal{T}_{n \times m}$ such that $\mathcal{P} \mathcal{T}_{n \times m}=\langle G, X\rangle$. The group $G$, being isomorphic to $\mathcal{S}_{n}\left\langle\mathcal{S}_{m}\right.$, is generated by 2 elements (Lemma 4.2(ii)), and hence, by Lemma 4.2(iii), it suffices to show that $|X| \geqslant 3$. Since the rank of a product is not greater than the minimum of the ranks of its factors and elements of rank $\mathrm{nm}$ form a subgroup, we obtain that $X$ must contain at least one element of rank $n m-1$.

We claim that $X$ must in fact contain at least two elements of rank $n m-1$, namely one strictly partial transformation, and one full transformation. Assume by contradiction that $X$ only contains one element, $\alpha$ say, with rank $n m-1$. Then an element $\beta \in \mathcal{P} \mathcal{T}_{n \times m}$ with rank $n m-1$ can be written as a product in $X$ and $\mathcal{S}_{n}$ < $\mathcal{S}_{m}$ and such a product cannot contain a transformation with rank less than $n m-1$. Hence $\beta$ must be written as a product in $\mathcal{S}_{n} \prec \mathcal{S}_{m}$ and $\alpha$. Now, if $\alpha$ were a strictly partial transformation, then no full transformation $\beta$ with rank $n m-1$ could be written in this form, which is a contradiction. Similarly, if $\alpha$ were a full transformation, then no strictly partial transformation with rank $n m-1$ could be written as a product in $\mathcal{S}_{n} \prec \mathcal{S}_{m}$ and $\alpha$. Thus $X$ must contain at least two elements with rank $n m-1$, namely one full and one strictly partial, as claimed.

Finally we prove that $X$ must contain a third element. Assume by contradiction that $X=\left\langle\alpha_{1}, \alpha_{2}\right\rangle$. We have, by the previous paragraph, that rank $\alpha_{1}=\operatorname{rank} \alpha_{2}=n m-1$ and we may assume without loss of generality that $\alpha_{1}$ is full and $\alpha_{2}$ is strictly partial. Let $\beta_{1} \in \mathcal{T}_{m}$ be an element of rank $m-1$ and set $\beta=\beta_{1} \bar{\varepsilon} \varphi$ where $\varphi: \mathcal{P} \mathcal{T}_{n} \curlywedge \mathcal{T}_{m} \rightarrow \mathcal{P} \mathcal{T}_{n \times m}$ is the homomorphism defined before Lemma 3.1. By Lemma 3.1, the elements $\alpha_{1}, \alpha_{2}$, and $\beta$ have unique preimages $\alpha_{1}^{\prime}, \alpha_{2}^{\prime}$, and $\beta^{\prime}$ in $\mathcal{P} \mathcal{T}_{n} \prec \mathcal{T}_{m}$, respectively, under the homomorphism $\varphi$. Since $\beta$ must be written as a word in $G, \alpha_{1}$, and $\alpha_{2}$, we have that $\beta^{\prime}$ must be written as a word in $\mathcal{S}_{n} \imath \mathcal{S}_{m}, \alpha_{1}^{\prime}$ and $\alpha_{2}^{\prime}$. However, the map $\alpha \mapsto \bar{\alpha}$, defined before Lemma 3.1, is a homomorphism $\mathcal{P} \mathcal{T}_{n} \imath \mathcal{T}_{m} \rightarrow \mathcal{T}_{m}$ by equation (2). As $\overline{\alpha_{1}^{\prime}}, \overline{\alpha_{2}^{\prime}} \in \mathcal{S}_{m}$, we obtain that $\overline{\beta^{\prime}} \in \mathcal{S}_{m}$. Since $\bar{\beta}^{\prime}=\beta_{1}$ and, by assumption, rank $\beta_{1}=m-1$, this is a contradiction. Thus $X$ must contain a third element as claimed.

The last lemma implies Theorem 1.1(ii). Lemma 4.2(ii) and the argument of the proof of Theorem 4.1 yield explicit generating sets for $\mathcal{P} \mathcal{T}_{n} \prec \mathcal{T}_{m}$ and $\mathcal{P} \mathcal{T}_{n \times m}$. Recall that the homomorphism $\varphi: \mathcal{P} \mathcal{T}_{n} \prec \mathcal{T}_{m} \rightarrow \mathcal{P} \mathcal{T}_{n \times m}$ was defined before Lemma 3.1.

Corollary 4.3. Let $\xi_{1}, \xi_{2}, \tau, \bar{\tau}$ be the elements of $\mathcal{T}_{n} \prec \mathcal{T}_{m}$ defined before Lemma 4.2 and let $\sigma$ be the element defined in the proof of Theorem 4.1. Then $\left\{\xi_{1}, \xi_{2}, \tau, \bar{\tau}, \sigma\right\}$ and $\left\{\xi_{1} \varphi, \xi_{2} \varphi, \tau \varphi, \bar{\tau} \varphi, \sigma \varphi\right\}$ are minimal generating sets for $\mathcal{P} \mathcal{T}_{n} \curlywedge \mathcal{T}_{m}$ and $\mathcal{P} \mathcal{T}_{m \times n}$ respectively.

\section{Presentations for $\mathcal{P} \mathcal{T}_{n} \prec \mathcal{T}_{m}$ and $\mathcal{P} \mathcal{T}_{n \times m}$}

Let $S$ be a monoid with generating set $X$ and let $X^{*}$ denote the free monoid on $X$. A word $w$ in $X$ can be considered as an element of $S$ and also as an element of $X^{*}$, but this will cause no confusion. Let $R$ denote the equivalence relation on $X^{*}$ defined by the rule that $\left(w_{1}, w_{2}\right) \in R$ if and only if $w_{1}=w_{2}$ holds in $S$. Then $R$ is in fact a congruence 
relation. Suppose, further, that $\mathcal{R} \subseteq X^{*} \times X^{*}$ such that the smallest congruence relation on $X^{*}$ containing $\mathcal{R}$ is $R$. In this case we say that $\mathcal{R}$ is a set of defining relations for $S$ with respect to the generating set $X$ and write that $S=\langle X \mid \mathcal{R}\rangle$. The pair $\langle X \mid \mathcal{R}\rangle$ is called a presentation for $S$. A relation $\left(w_{1}, w_{2}\right) \in \mathcal{R}$ is usually written as $w_{1}=w_{2}$.

Let $n, m \geqslant 2$. To discuss presentations of $\mathcal{P} \mathcal{T}_{n} \prec \mathcal{T}_{m}$ and $\mathcal{P} \mathcal{T}_{n \times m}$ let us fix generators of these monoids. As in Section 3 , for $i \in \underline{m}, \varepsilon^{(i)}$ denotes the $i$-th coordinate embedding $\mathcal{P} \mathcal{T}_{n} \rightarrow \mathcal{P} \mathcal{T}_{n} \prec \mathcal{T}_{m}$ and $\bar{\varepsilon}$ denotes the embedding $\mathcal{T}_{m} \rightarrow \mathcal{P} \mathcal{T}_{n} \prec \mathcal{T}_{m}$ into the $(m+1)$-th component. Let us consider the following elements of $\mathcal{P} \mathcal{T}_{n} \prec \mathcal{T}_{m}$ :

$$
\begin{aligned}
\pi & =(12) \varepsilon^{(1)} \\
\varrho & =(12 \ldots n) \varepsilon^{(1)} \\
\tau & =[2,2,3,4, \ldots, n] \varepsilon^{(1)} \\
\sigma & =[\varnothing, 2,3, \ldots, n] \varepsilon^{(1)} \\
\bar{\pi} & =(12) \bar{\varepsilon} ; \\
\bar{\varrho} & =(12 \ldots m) \bar{\varepsilon} ; \\
\bar{\tau} & =[2,2,3,4, \ldots, m] \bar{\varepsilon} .
\end{aligned}
$$

We have that $\langle\pi, \varrho, \tau, \sigma\rangle \cong \mathcal{P} \mathcal{T}_{n}$ under the map $\varepsilon^{(1)}$ and that $\langle\bar{\pi}, \bar{\varrho}, \bar{\tau}\rangle \cong \mathcal{T}_{m}$ under the embedding $\bar{\varepsilon}$. Hence we may identify $\mathcal{P} \mathcal{T}_{n}$ with $\langle\pi, \varrho, \tau, \sigma\rangle$ and $\mathcal{T}_{m}$ with $\langle\bar{\pi}, \bar{\varrho}, \bar{\tau}\rangle$. Let $\mathcal{R}_{P}$ and $\mathcal{R}_{T}$ be sets of defining relations for $\mathcal{P} \mathcal{T}_{n}$ and $\mathcal{T}_{m}$ with respect to the generating sets $\{\pi, \varrho, \tau, \sigma\}$ and $\{\bar{\pi}, \bar{\varrho}, \bar{\tau}\}$, respectively. Explicit expressions for $\mathcal{R}_{T}$ and $\mathcal{R}_{P}$ can be found in [Fer02, Section 2].

Let $S$ and $Q$ be two monoids defined by the presentations $\left\langle X_{S} \mid \mathcal{R}_{S}\right\rangle$ and $\left\langle X_{Q} \mid \mathcal{R}_{Q}\right\rangle$, respectively. Let $S \rtimes Q$ be a semidirect product of $S$ by $Q$ with respect to a left action of $Q$ on $S$. For each $a \in X_{S}$ and $b \in X_{Q}$, denote by ${ }^{b} a$ a (fixed) word of $X_{S}^{*}$ that represents the image of $a$ under $b$ in $S$. Then Lavers proved in [Lav98, Corollary 2] (see also [Ara01]) that

$$
S \rtimes Q=\left\langle X_{S} \cup X_{Q} \mid \mathcal{R}_{S} \cup \mathcal{R}_{Q} \cup\left\{b a=\left({ }^{b} a\right) b \mid a \in X_{S}, b \in X_{Q}\right\}\right\rangle .
$$

Using this result in the case when the left action is trivial, we obtain that

$$
S \times Q=\left\langle X_{S} \cup X_{Q} \mid \mathcal{R}_{S} \cup \mathcal{R}_{Q} \cup\left\{b a=a b \mid a \in X_{S}, b \in X_{Q}\right\}\right\rangle .
$$

Next define the following sets of relations in $\{\pi, \varrho, \tau, \sigma, \bar{\pi}, \bar{\varrho}, \bar{\tau}\}$ :

$$
\begin{aligned}
\mathcal{R}_{1}= & \left\{\bar{\varrho}^{m-j+1} u \bar{\varrho}^{m+j-k} v \bar{\varrho}^{k-1}=\bar{\varrho}^{m-k+1} v \bar{\varrho}^{m+k-j} u \bar{\varrho}^{j-1} \mid j, k \in \underline{m}, j<k, u, v \in\{\pi, \varrho, \tau, \sigma\}\right\} ; \\
\mathcal{R}_{2}= & \left\{\bar{\pi} \varrho^{m-1} u \varrho=u \bar{\pi}, \bar{\pi} \bar{\varrho}^{m-j+1} u \bar{\varrho}^{j-1}=\bar{\varrho}^{m-j+1} u \bar{\varrho}^{j-1} \bar{\pi} \mid 3 \leqslant j \leqslant m, u \in\{\pi, \varrho, \tau, \sigma\}\right\} ; \\
\mathcal{R}_{3}= & \left\{\bar{\tau} u=\bar{\tau}, \bar{\tau} \bar{\varrho}^{m-1} u \varrho=u \bar{\varrho}^{m-1} u \overline{\varrho \tau}, \bar{\tau}^{m-j+1} u \bar{\varrho}^{j-1}=\bar{\varrho}^{m-j+1} u \bar{\varrho}^{j-1} \bar{\tau} \mid\right. \\
& 3 \leqslant j \leqslant m, u \in\{\pi, \varrho, \tau, \sigma\}\} .
\end{aligned}
$$


The next result states presentations for $\mathcal{P} \mathcal{T}_{n} \prec \mathcal{T}_{m}$ and for $\mathcal{P} \mathcal{T}_{n \times m}$ with respect to the generating sets $\{\pi, \varrho, \tau, \sigma, \bar{\pi}, \bar{\varrho}, \bar{\tau}\}$ and $\{\pi \varphi, \varrho \varphi, \tau \varphi, \sigma \varphi, \bar{\pi} \varphi, \bar{\varrho} \varphi, \bar{\tau} \varphi\}$, respectively, where $\varphi: \mathcal{P} \mathcal{T}_{n} \prec \mathcal{T}_{m} \rightarrow \mathcal{P} \mathcal{T}_{n \times m}$ is the epimorphism defined before Lemma 3.1. To simplify notation, we will omit " $\varphi$ " from the description of the elements of $\mathcal{P} \mathcal{T}_{n \times m}$, and hence we will consider an element of $\mathcal{P} \mathcal{T}_{n} \prec \mathcal{T}_{m}$ as an element of $\mathcal{P} \mathcal{T}_{n \times m}$.

Theorem 5.1. (i) $\mathcal{P} \mathcal{T}_{n}\left\langle\mathcal{T}_{m}=\left\langle\pi, \varrho, \tau, \sigma, \bar{\pi}, \bar{\varrho}, \bar{\tau} \mid \mathcal{R}_{P} \cup \mathcal{R}_{T} \cup \mathcal{R}_{1} \cup \mathcal{R}_{2} \cup \mathcal{R}_{3}\right\rangle\right.$;

(ii) $\mathcal{P} \mathcal{T}_{n \times m}=\left\langle\pi, \varrho, \tau, \sigma, \bar{\pi}, \varrho, \bar{\tau} \mid \mathcal{R}_{P} \cup \mathcal{R}_{T} \cup \mathcal{R}_{1} \cup \mathcal{R}_{2} \cup \mathcal{R}_{3} \cup\left\{(\varrho \sigma)^{n}=(\varrho \sigma)^{n} \bar{\tau}\right\}\right\rangle$.

Proof. (i) By the definition of the wreath product,

$$
\mathcal{P} \mathcal{T}_{n} \prec \mathcal{T}_{m}=\left(\left(\mathcal{P} \mathcal{T}_{n}\right) \varepsilon^{(1)} \times \cdots \times\left(\mathcal{P} \mathcal{T}_{n}\right) \varepsilon^{(m)}\right) \rtimes\left(\mathcal{T}_{m} \bar{\varepsilon}\right)
$$

For $i \in\{1, \ldots, m\}$ set $\pi_{i}, \varrho_{i}, \tau_{i}$ and $\sigma_{i}$ as $(12) \varepsilon^{(i)},(12 \ldots n) \varepsilon^{(i)},[2,2,3,4, \ldots, n] \varepsilon^{(i)}$, and $[\varnothing, 2,3, \ldots, n] \varepsilon^{(i)}$, respectively. Set $\mathcal{G}_{i}=\left\{\pi_{i}, \varrho_{i}, \tau_{i}, \sigma_{i}\right\}$ and $\overline{\mathcal{G}}=\{\bar{\pi}, \bar{\varrho}, \bar{\tau}\}$. Then, by (77), the set $\mathcal{G}_{1} \cup \cdots \cup \mathcal{G}_{m} \cup \overline{\mathcal{G}}$ is a generating set of $\mathcal{P} \mathcal{T}_{n} \prec \mathcal{T}_{m}$ and combining (5), (6) and the definition of the left action of $\mathcal{T}_{m}$ on $\left(\mathcal{P} \mathcal{T}_{n}\right)^{m}$ given in Section 2 , we obtain that

$$
\mathcal{P} \mathcal{T}_{n} \prec \mathcal{T}_{m}=\left\langle\mathcal{G}_{1} \cup \cdots \cup \mathcal{G}_{m} \cup \overline{\mathcal{G}} \mid \mathcal{R}_{1 P} \cup \cdots \cup \mathcal{R}_{m P} \cup \mathcal{R}_{T} \cup \mathcal{R}\right\rangle
$$

where, for $i \in\{1, \ldots, m\}$ the set $\mathcal{R}_{i P}$ is the set of relations that we obtain from $\mathcal{R}_{P}$ by substituting $\pi_{i}, \varrho_{i}, \tau_{i}$ and $\sigma_{i}$ in the places of $\pi, \varrho, \tau$ and $\sigma$, respectively. Moreover, $\mathcal{R}$ is the set consisting of the following relations:

$$
\begin{aligned}
& u v=v u \quad u \in \mathcal{G}_{i}, v \in \mathcal{G}_{j} \text { with } i<j \text {; } \\
& \bar{\pi} \pi_{1}=\pi_{2} \bar{\pi}, \quad \bar{\pi} \varrho_{1}=\varrho_{2} \bar{\pi}, \quad \bar{\pi} \tau_{1}=\tau_{2} \bar{\pi}, \quad \bar{\pi} \sigma_{1}=\sigma_{2} \bar{\pi} ; \\
& \bar{\pi} \pi_{2}=\pi_{1} \bar{\pi}, \quad \bar{\pi} \varrho_{2}=\varrho_{1} \bar{\pi}, \quad \bar{\pi} \tau_{2}=\tau_{1} \bar{\pi}, \quad \bar{\pi} \sigma_{2}=\sigma_{1} \bar{\pi} ; \\
& \bar{\pi} \pi_{i}=\pi_{i} \bar{\pi}, \quad \bar{\pi} \varrho_{i}=\varrho_{i} \bar{\pi}, \quad \bar{\pi} \tau_{i}=\tau_{i} \bar{\pi}, \quad \bar{\pi} \sigma_{i}=\sigma_{i} \bar{\pi} \quad 3 \leqslant i \leqslant m ; \\
& \bar{\varrho} \pi_{i}=\pi_{i-1} \bar{\varrho}, \quad \bar{\varrho} \varrho_{i}=\varrho_{i-1} \bar{\varrho}, \quad \bar{\varrho} \tau_{i}=\tau_{i-1} \bar{\varrho}, \quad \bar{\varrho} \sigma_{i}=\sigma_{i-1} \bar{\varrho} \quad 2 \leqslant i \leqslant m \text {; } \\
& \bar{\varrho} \pi_{1}=\pi_{m} \bar{\varrho}, \quad \bar{\varrho} \varrho_{1}=\varrho_{m} \bar{\varrho}, \quad \bar{\varrho} \tau_{1}=\tau_{m} \bar{\varrho}, \quad \bar{\varrho} \sigma_{1}=\sigma_{m} \bar{\varrho} ; \\
& \bar{\tau} \pi_{1}=\bar{\tau}, \quad \bar{\tau} \varrho_{1}=\bar{\tau}, \quad \bar{\tau} \tau_{1}=\bar{\tau}, \quad \bar{\tau} \sigma_{1}=\bar{\tau} ; \\
& \bar{\tau} \pi_{2}=\pi_{1} \pi_{1} \bar{\tau}, \quad \bar{\tau} \varrho_{2}=\varrho_{1} \varrho_{2} \bar{\tau}, \quad \bar{\tau} \tau_{2}=\tau_{1} \tau_{2} \bar{\tau}, \quad \bar{\tau} \sigma_{1}=\sigma_{1} \sigma_{2} \bar{\tau} \\
& \bar{\tau} \pi_{i}=\pi_{i} \bar{\tau}, \quad \bar{\tau} \varrho_{i}=\varrho_{i} \bar{\tau}, \quad \bar{\tau} \tau_{i}=\tau_{i} \bar{\tau}, \quad \bar{\tau} \sigma_{i}=\sigma_{i} \bar{\tau} \quad 3 \leqslant i \leqslant m .
\end{aligned}
$$

Note that $\pi_{1}=\pi, \varrho_{1}=\varrho, \tau_{1}=\tau$, and $\sigma_{1}=\sigma$. Further, $i \geqslant 2$, we have that $\pi_{i}=\bar{\varrho}^{m-i+1} \pi \varrho^{i-1}$ and we have analogous expressions for $\varrho_{i}, \tau_{i}$, and $\sigma_{i}$. Hence for $i \geqslant 2$, we can remove the elements of $\mathcal{G}_{i}$ from the generating set given in (8) and we can also remove the relation sets $\mathcal{R}_{i P}$. Further we replace the occurrences in $\mathcal{R}$ of the generators belonging to $\mathcal{G}_{i}$ by the corresponding expressions in $\pi, \varrho, \tau$ and $\sigma$. This way the relations in (13)-(14) will transform into the trivial relation. As $\pi^{2}=1$ follows from the relations in $\mathcal{R}_{P}$, the relations in (11) can be removed, as they follow from the relations in (10). This way, the relations in (9) lead to the relations in $\mathcal{R}_{1}$, the relations in (10) and (12) 
give the relations in $\mathcal{R}_{2}$, and finally the relations in (15)-(17) will result in the relation set $\mathcal{R}_{3}$. This shows that the presentation in part (i) is valid.

(ii) As $(\varrho \sigma)^{n}=\varnothing$, the second statement follows from part (i) and Lemma 3.1(iii).

Theorem 5.1 gives a presentation of $\mathcal{P} \mathcal{T}_{n} \imath \mathcal{T}_{m}$ and $\mathcal{P} \mathcal{T}_{n \times m}$ in terms of 7 generators, even though we proved in Theorem 4.1 that these monoids are generated by 5 generators. In order to pass to the 5 -element generating set, we replace the 4-generator set $\{\pi, \varrho, \bar{\pi}, \bar{\varrho}\}$ of $\mathcal{S}_{n}\left\{\mathcal{S}_{m}\right.$ used in Theorem 5.1 with the generating set $\left\{\xi_{1}, \xi_{2}\right\}$ given at the beginning of Section 4. In order two write down a presentation that is satisfied by the generating set $\left\{\xi_{1}, \xi_{2}, \tau, \sigma, \bar{\tau}\right\}$, we need to express the generators $\pi, \varrho, \bar{\pi}, \bar{\varrho}$ in terms of the generators $\xi_{1}$ and $\xi_{2}$. We do this in the next lemma, whose proof, though rather technical, is routine, and so we omit the details. We note that the following lemma also implies [AS09, Theorem 4.1] that $\mathcal{S}_{n} \prec \mathcal{S}_{m}$ is generated by two elements.

Lemma 5.2. Let $m, n \geqslant 2$. If $m$ and $n$ are both even then

$$
\begin{aligned}
& \pi=\left(\xi_{1}^{m-1} \xi_{2}^{2}\right)^{(m-2)(n-1)^{2}}\left(\xi_{1} \xi_{2}^{2}\right)^{(m-1)\left(n^{2}-n-1\right)}\left(\xi_{1} \xi_{2}\right)^{m} ; \\
& \varrho=\left(\left(\xi_{1}^{m-1} \xi_{2}^{2}\right)^{(m-2)(n-1)^{2}}\left(\xi_{1} \xi_{2}^{2}\right)^{(m-1)\left(n^{2}-n-1\right)}\right)^{n-1} ; \\
& \bar{\pi}=\xi_{2}^{2 n-1}\left(\left(\xi_{1}^{m-1} \xi_{2}^{2}\right)^{(m-2)(n-1)^{2}}\left(\xi_{1} \xi_{2}^{2}\right)^{(m-1)\left(n^{2}-n-1\right)}\right)^{n-1} ; \\
& \bar{\varrho}=\xi_{2}^{2 n-1}\left(\left(\xi_{1}^{m-1} \xi_{2}^{2}\right)^{(m-2)(n-1)^{2}}\left(\xi_{1} \xi_{2}^{2}\right)^{(m-1)\left(n^{2}-n-1\right)}\right)^{n}\left(\xi_{1} \xi_{2}\right)^{m} \xi_{2}^{2 n-1} \xi_{1} \xi_{2} .
\end{aligned}
$$

If either $m$ or $n$ is odd then

$$
\begin{aligned}
\pi & =\left(\xi_{1} \xi_{2}^{2 n-1}\right)^{(m-1) n}\left(\xi_{1}^{m+1} \xi_{2}^{2 n-1}\right)^{(m-1)(n-1)} \xi_{1}^{m} ; \\
\varrho & =\left(\xi_{1} \xi_{2}^{2 n-1}\right)^{n-1}\left(\xi_{1}^{m+1} \xi_{2}^{2 n-1}\right)^{(m-2)(n-1)} ; \\
\bar{\pi} & =\xi_{1}^{2 m-1}\left(\xi_{1} \xi_{2}^{2 n-1}\right)^{n}\left(\xi_{1}^{m+1} \xi_{2}^{2 n-1}\right)^{(m-2)(n-1)} ; \\
\bar{\varrho} & =\xi_{1}^{2 m-1}\left(\xi_{1} \xi_{2}^{2 n-1}\right)^{(m-1) n}\left(\xi_{1}^{m+1} \xi_{2}^{2 n-1}\right)^{(m-1)(n-1)} \xi_{1}^{m+2} .
\end{aligned}
$$

Suppose that $w_{1}, w_{2}, w_{3}$ and $w_{4}$ are the words on the right hand sides of the equations (18)-(21), when both $m$ and $n$ are even, or of the equations (22)-(25) otherwise, respectively. Let $\overline{\mathcal{R}}_{P}, \overline{\mathcal{R}}_{T}, \overline{\mathcal{R}}_{1}, \overline{\mathcal{R}}_{2}$ and $\overline{\mathcal{R}}_{3}$ the sets of relations that are constructed by substituting in $\mathcal{R}_{P}, \mathcal{R}_{T}, \mathcal{R}_{1}, \mathcal{R}_{2}$ and $\mathcal{R}_{3}$ the words $w_{1}, w_{2}, w_{3}, w_{4}$ in the places of $\pi, \varrho, \bar{\pi}$, $\bar{\varrho}$, respectively. Further, let $\bar{r}$ be the relation obtained by substituting $w_{2}$ in the relation $(\varrho \sigma)^{n}=(\varrho \sigma)^{n} \bar{\tau}$ into the place of $\varrho$. Then we obtain the following main result of this section.

Corollary 5.3. (i) $\mathcal{P} \mathcal{T}_{n} \curlywedge \mathcal{T}_{m}=\left\langle\xi_{1}, \xi_{2}, \tau, \sigma, \bar{\tau} \mid \overline{\mathcal{R}}_{P} \cup \overline{\mathcal{R}}_{T} \cup \overline{\mathcal{R}}_{1} \cup \overline{\mathcal{R}}_{2} \cup \overline{\mathcal{R}}_{3}\right\rangle$;

(ii) $\mathcal{P} \mathcal{T}_{n \times m}=\left\langle\xi_{1}, \xi_{2}, \tau, \sigma, \bar{\tau} \mid \overline{\mathcal{R}}_{P} \cup \overline{\mathcal{R}}_{T} \cup \overline{\mathcal{R}}_{1} \cup \overline{\mathcal{R}}_{2} \cup \overline{\mathcal{R}}_{3} \cup\{\bar{r}\}\right\rangle$. 


\section{ACKNOWLEDGEMENT}

Cicalò gratefully acknowledges the support of FCT and PIDDAC, within the project PTDC/MAT/69514/2006 of CAUL and the support of the Regione Sardegna, within the project Master \& Back, PR-MAB-A2009-837. She also thanks the Department of Mathematics of the University of Trento for its hospitality from 2011. Fernandes gratefully acknowledges support of FCT and PIDDAC, within the projects ISFL-1-143 and PTDC/MAT/69514/2006 of CAUL. Schneider was supported by the FCT project PTDC/MAT/101993/2008.

\section{REFERENCES}

[Ă̌z58] A. Ja. Aǔzenštat. Defining relations of finite symmetric semigroups. Mat. Sb. N.S., 45 (87):261$280,1958$.

[Ara01] Isabel M. Araújo. Presentations for semigroup constructions and related computational methods. PhD thesis, University of St Andrews, 2001.

[AS09] João Araújo and Csaba Schneider. The rank of the endomorphism monoid of a uniform partition. Semigroup Forum, 78(3):498-510, 2009.

[Eas06] James East. A presentation of the singular part of the symmetric inverse monoid. Comm. Algebra, 34(5):1671-1689, 2006.

[Eas10a] James East. A presentation for the singular part of the full transformation semigroup. Semigroup Forum, 81(2):357-379, 2010.

[Eas10b] James East. Presentations for singular subsemigroups of the partial transformation semigroup. Internat. J. Algebra Comput., 20(1):1-25, 2010.

[Eas11a] James East. Generators and relations for partition monoids and algebras. J. Algebra, 339:1-26, 2011.

[Eas11b] James East. On the singular part of the partition monoid. Internat. J. Algebra Comput., 21(12):147-178, 2011.

[EEF08] David Easdown, James East, and D. G. FitzGerald. A presentation of the dual symmetric inverse monoid. Internat. J. Algebra Comput., 18(2):357-374, 2008.

[Fer02] Vítor H. Fernandes. Presentations for some monoids of partial transformations on a finite chain: a survey. In Semigroups, algorithms, automata and languages (Coimbra, 2001), pages 363-378. World Sci. Publ., River Edge, NJ, 2002.

[FQ] Vítor H. Fernandes and Teresa M. Quinteiro. On the ranks of certain monoids of transformations that preserve a uniform partition. submitted.

[FQ11] Vítor H. Fernandes and Teresa M. Quinteiro. On the monoids of transformations that preserve the order and a uniform partition. Comm. Algebra, 39(8):2798-2815, 2011.

[FQ12] Vítor H. Fernandes and Teresa M. Quinteiro. The cardinal of various monoids of transformations that preserve a uniform partition. Bull. Malays. Math. Sci. Soc. (2), 35(4):885-896, 2012.

[GM09] Olexandr Ganyushkin and Volodymyr Mazorchuk. Classical finite transformation semigroups, volume 9 of Algebra and Applications. Springer-Verlag London Ltd., London, 2009. An introduction.

[HD05] Pei Huisheng and Zou Dingyu. Green's equivalences on semigroups of transformations preserving order and an equivalence relation. Semigroup Forum, 71(2):241-251, 2005.

[Hui05] Pei Huisheng. On the rank of the semigroup $T_{E}(X)$. Semigroup Forum, 70(1):107-117, 2005.

[Lav98] T. G. Lavers. Presentations of general products of monoids. J. Algebra, 204(2):733-741, 1998. 
[Moo96] Eliakim Hastings Moore. Concerning the abstract groups of order $k$ ! and $\frac{1}{2} k$ ! holohedrically isomorphic with the symmetric and the alternating substitution-groups on $k$ letters. Proc. London Math. Soc., 28(1):357-367, 1896.

[Pop62] L. M. Popova. Defining relations of a semigroup of partial endomorphisms of a finite linearly ordered set. Leningrad. Gos. Ped. Inst. Učen. Zap., 238:78-88, 1962.

[RS09] John Rhodes and Benjamin Steinberg. The q-theory of finite semigroups. Springer Monographs in Mathematics. Springer, New York, 2009.

[Ruš95] Nikola Ruškuc. Semigroup presentations. PhD thesis, University of St Andrews, 1995.

[SPC07] Lei Sun, Huisheng Pei, and Zhengxing Cheng. Regularity and Green's relations for semigroups of transformations preserving orientation and an equivalence. Semigroup Forum, 74(3):473-486, 2007.

(Cicalò) Università degli Studi di Cagliari, Dipartimento di Matematica e Informatica, Via Ospedale, 72 - 09124 Cagliari, Italy; E-Mail: CiCAlo@SCIENCE.Unitn.it

(Fernandes) Departamento de Matemática, Faculdade de Ciências e Techologia, Universidade Nova de Lisboa, Monte da Caparica, 2829-516 Caparica, Portugal; also: Centro de Álgebra da Universidade de Lisboa, Av. Prof. Gama Pinto 2, 1649-003 Lisboa, Portugal; E-MAIL:VHF@FCT.UNL.PT

(Schneider) Centro de Álgebra da Universidade de Lisboa, Av. Prof. Gama Pinto 2, 1649-003 Lisboa, Portugal; E-MaIL: CSABA.SChNEIDER@GMail.Com 